\title{
OS SISTEMAS DE INFORMAÇÃO GEOGRÁFICA E O PLANEJAMENTO URBANO SAUDÁVEL NA AMADORA
}

\author{
Paula Santana ${ }^{1}$ \\ Cláudia Costa ${ }^{2}$ \\ Adriana Loureiro ${ }^{3}$
}

Resumo: A saúde é um tema estratégico para o desenvolvimento nacional, pelo que a intervenção na sociedade apenas pode ser sustentada e efetiva se resultar de informação fidedigna, fiável, adequada e disponibilizada em tempo útil. Para isso têm contribuído os Sistemas de Informação Geográfica (SIG). Será apresentado um trabalho relativo à influência do contexto na saúde da população do Município de Amadora, ilustrando a aplicabilidade do SIG a uma escala de análise local, com o objetivo de construir indicadores, que permitam conhecer o território, as suas potencialidades e os seus constrangimentos, aplicáveis no planeamento urbano saudável.

Palavras-chave: Planejamento Urbano Saudável; Sistemas de Informação Geográfica; Resultados em Saúde; Políticas de Saúde; Indicadores.

\section{The geographic information systems and the healthy urban planning in Amadora}

\begin{abstract}
Health is a strategic theme for national development; therefore intervention in society can only be sustained and effective if it is a result of trustworthy, reliable, adequate
\end{abstract}

\footnotetext{
${ }^{1}$ Geógrafa. Doutorada em Geografia e Professora Catedrática da Universidade de Coimbra. Tem-se dedicado à docência e investigação da geografia da saúde e do planeamento urbano saudável, com destaque para a associação entre os aspectos de contexto e de composição e a saúde e bem-estar da população. A relevância destes temas tem-se traduzido em conferências e comunicações proferidas em todo o Mundo, em aulas que lecionou em Portugal (em diversos cursos e instituições) e no Estrangeiro (Reino Unido, Espanha, Finlândia, Macau e Brasil), em 4 prémios de reconhecimento científico que já ganhou e em publicações - 13 livros e mais de 200 títulos em revistas nacionais e internacionais. Coordena vários projectos de âmbito nacional (financiados pela Fundação para a Ciência e Tecnologia e Ministério da Saúde). É referee de 7 importantes revistas internacionais e de programas científicos (European Science Foundation e Organização Mundial de Saúde). Membro Correspondente da Geographical Society of Finland, eda Commission on Health and Development da União Internacional de GeografialGU). Representa(ou) Portugal em vários programas científicos internacionais (financiados, entre outras, pela Organização Mundial de Saúde, União Europeia, European Science Foundation e o Medical Research Council de Glasgow). Cumpriu funções como Vice-Presidente da Comissão de Coordenação e Desenvolvimento Regional de Lisboa e Vale do Tejo (CCDR-LVT) entre Janeiro de 2009 eJaneiro de 2012.

${ }^{2}$ Geógrafa. Doutoranda em Geografia na Universidade de Coimbra. Mestre em Ciência \& Sistemas de Informação Geográfica no ISEGI-UNL (2011), com a dissertação "Localização óptima do futuro Hospital de Sintra: aplicação de modelos de location-allocation no planeamento de cuidados de saúde". Pós-graduada em Sistemas de Informação Geográfica pela Universidade Atlântica (2005). Licenciada em Geografia, Ordenamento do Território e Desenvolvimento pela Universidade de Coimbra (2003). Estudante Erasmus na Universidade de Utrecht (Holanda) (2002). Membro da equipa do Grupo de Investigação em Geografia da Saúde, Centro de Estudos em Geografia e Ordenamento do Território, na Universidade de Coimbra, desde 2008. As suas áreas de investigação têm passado pelo desenvolvimento e implementação de projetos de Sistemas de Informação Geográfica aplicados ao planeamento em saúde de recursos humanos e equipamentos e à avaliação quer das condições de contexto em que a população reside, quer da distribuição de resultados em saúde.

${ }^{3}$ Geógrafa. Doutoranda em Geografia na Universidade de Coimbra. Mestre em Geografia Humana, Ordenamento do Território e Desenvolvimento pela Universidade de Coimbra (2011), com a dissertação "Abordagem Epidemiológica da Utilização do Hospital Professor Doutor Fernando Fonseca segundo os Grupos de Diagnósticos Homogéneos". Bolseira SYLFF (2011). Licenciada em Geografia, Ordenamento do Território e Desenvolvimento pela Universidade de Coimbra (2007). Estudante Erasmus na Universidade Complutense de Madrid (2006). Membro da equipa do Grupo de Investigação em Geografia da Saúde, Centro de Estudos em Geografia e Ordenamento do Território, na Universidade de Coimbra, desde 2008. Técnica Superior do programa municipal "Figueira, Cidade Saudável" da Câmara Municipal da Figueira da Foz, entre 2011 e 2012. Tem participado em projetos de planeamento e caraterização em saúde, nomeadamente ao nível do planeamento urbano saudável e da avaliação de impacto em saúde. Actualmente é bolseira de doutoramento da FCT e investigadora do Projeto SMAILE - Saúde Mental - Avaliação do Impacte das Condicionantes Locais e Económicas, financiado pela Fundação para a Ciência e Tecnologia (PTDC/ATP-GEO/4101/2012), desde 2013.
} 
and timely information. And that is what Geographic Information Systems (GIS) have contributed for. A work will be presented regarding the influence of the context on population's health (municipality of Amadora), thus illustrating the applicability of GIS to a local analysis scale, with the purpose of building indicators which allow us to know the territory, its potentials and its limitations, applicable in healthy urban planning.

Keywords: Healthy Urban Planning; Geographic Information Systems; Results in Health; Health Policies; Indicators.

\section{INTRODUÇÃO}

No início do século XXI, algumas das maiores questões políticas e académicas prendem-se com o ambiente, a sustentabilidade, a qualidade de vida urbana e a saúde. Estas preocupações são também o resultado da progressiva urbanização do espaço e da aparente contradição entre o papel historicamente desempenhado pelas cidades, enquanto criadoras de riqueza e geradoras de bem-estar e a atual realidade urbana de pobreza, insegurança e anomia(SANTANA, 2009).

Nesse sentido, o espaço urbano não encontra mais o seu propósito fechado nos aspectos tradicionais, eminentemente físicos (traçado de vias públicas e meios de comunicação, orientação da composição arquitetônica e das edificações, entre outras), pois cada vez mais tem de ter em conta não só as dimensões imateriais da cidade (percepção da segurança, sentido de pertença, confiança, etc.) mas também a necessidade de se associar a valores como qualidade de vida, bem-estar e saúde das populações, procurando dar resposta a problemas de saúde pública evidenciados na cidade (BARTON, TSOUROU, 2000; KEMM, 2006).

A saúde associada ao planejamento urbano tem ganho cada vez mais espaço nos fóruns de discussão académica e política. SANTANA(2005) refere que a influência do contexto na saúde é complexa e holística, porque o contexto é formado por múltiplos ambientes, dos quais se podem destacar o ambiente físico, o social, o económico e o cultural. Estes ambientes desdobram-se em diversos fatores, de que se destacam a poluição atmosférica, a qualidade da água, o capital social ou as oportunidades locais (bens e serviços). Planear lugares mais saudáveis, capazes de promover a saúde e a qualidade de vida dos seus habitantes é não esquecer nenhuma destas dimensões. Planear é identificar com precisão as 
características ambientais que, potencialmente, determinam o bem-estar e a qualidade de vida humana. De acordo com WEINSTEIN(1980) "we must fit the city to man's needs rather than fit man to the city's needs" para que viver na cidade seja um fator de aperfeiçoamento e não de degradação da vida humana. Por exemplo, a proximidade dos espaços verdes às áreas residenciais tem impactos indiretos na saúde, melhorando a qualidade do ar, atenuando o efeito da poluição e a "ilha de calor urbano" (WHITFORD et al., 2001; ALCOFORADO, ANDRADE, 2007; VASCONCELOS, VIEIRA, 2007) e proporcionando aos residentes um ambiente físico que incentiva a prática de atividade física, incluindo caminhar. A tarefa de identificar com precisão as características ambientais que podem influenciar a vida humana e a forma como podem ser medidas e monitorizadas, é um dos grandes desafios, dado que esse conhecimento é fundamental ao desenvolvimento de intervenções de planejamento saudável (SANTANA, 2009). Neste sentido, o uso de indicadores apropriados (materiais e imateriais) tem sido considerado um elemento central na operacionalização do conceito de cidade saudável.

É neste sentido que investigadores começam a implementar metodologiasSistemas de Informação Geográfica (SIG) que permitem identificar os fatores de contexto que têm maior impacto no bem-estar e na saúde individual e comunitária, para além da sua medição, avaliação e monitorização. Uma das virtudes dos SIG é a sua capacidade de cruzamento de informação de diversas fontes que têm em comum a localização geográfica (MEDRONHO, 1995). Assim, ao sustentar uma caracterização completa das entidades geográficas do espaço que se pretende analisar, constituem-se como o ambiente ideal para a implementação de metodologias SIG num contexto de planejamentourbano saudável (RIBEIRO, 2001). Adicionalmente, devido quer à capacidade de cruzamento de informação e à consideração de diferentes escalas quer ao facto de permitir análises espaciais e temporais fundamentais tanto na avaliação como na explicação de padrões e tendências de procura/utilização, são uma ferramenta importante na identificação de indicadores de contexto (ROCHA et al., 2000).

Em 2009 a Direção Geral da Saúde de Portugal preparou uma circular com recomendações para a integração e apreciação da componente Saúde Humana nos Planos Municipais de Ordenamento do Território (Circular informativa no 36/DA de 9 de Outubro de 2009). Este documento reconhece a importância da saúde no âmbito da questão ambiental e reforça a 
necessidade da temática "Saúde" nos instrumentos de ordenamento do território ir além dos aspetos relacionados com a rede de equipamentos de Saúde. De facto, os efeitos do ambiente envolvente na saúde humana são habitualmente descurados nos instrumentos de planejamento e gestão territorial, quando na verdade deveriam ser realçados, evidenciando a sua relevância e inter-relação, principalmente à escala municipal. As vantagens desta abordagem passam por: 1. a inclusão da saúde na previsão de impactos e prevenção deproblemas em fases posteriores; 2. a saúde é um elemento necessário para a sustentabilidade; 3. a saúde é uma preocupação fundamental da população envolvida; e 4. os ganhos em saúde constituem uma poderosa vantagem política (PARTIDÁRIO, JESUS, 2003). Apesar disso, o documento refere a ausência de metodologias apropriadas para o seu devido aprofundamento, tanto mais que alguns dos determinantes em saúde apresentam uma elevada subjetividade e são de apreciação complexa.

A utilização dos SIG são indispensáveis para responder a esta orientação do Ministério da Saúde em Portugal. Todavia, é importante reconhecer que existem limitações e constrangimentos à utilização dos SIG derivados, por um lado, da qualidade da informação (numérica e alfanumérica) e, por outro, da dificuldade de encontrar indicadores que permitam, realmente, medir a saúde. Por isso, colocam-se alguns desafios: 1. desenvolver sistemas integrados de recolha (seleção) de informação que permitam a análise da informação e verificação dos ganhos temporais e espaciais em saúde; 2.criar um sistema de difusão da informação e participação que utilize diferentes canais e chegue a diferentes atores (políticos, gestores, prestadores, sociedade civil e cidadãos) (SANTANA, 2005).

\section{O papel dos Sistemas de Informação Geográfica no Planejamento Urbano Saudável}

Saber interpretar, correlacionar e avaliar as características de um lugar (que se podem transformar em fatores de um determinado fenómeno) é de extrema importância, nomeadamente no que diz respeito à avaliação de impactos de políticas públicas que actuam sobre a cidade. Por isso, o planejamento urbano dispõe de um instrumento de trabalho privilegiado - os SIG. Esta ferramenta possibilita o mapeamento socioambiental, permitindo, deste modo, levar a cabo análises da cidade como um todo, ao mesmo tempo que facilitam a avaliação e intervenção na saúde urbana (BORGES, 2000). Trata-se de um 
sistema que integra a aquisição, o armazenamento, a análise (estatística e de modelos espaciais) e a apresentação de resultados através da representação em gráficos e mapas de dados geográficos relativos a referências espaciais associadas aos fenómenos que estão a ser avaliados. Assim, os SIG são uma ferramenta que, usando informação disponível, simula as actividades inteligentes do homem no estudo do território e na procura das melhores soluções para os problemas, constituindo uma ferramenta essencial para a tomada de decisão (SANTANA, 2005; ALMEIDAet al., 2007). As suas capacidades de inter-relação, interacção e integração de informação geográfica de variada natureza, fonte e formato são apontadas por muitos como um dos principais benefícios da sua utilização (BARCELLOS, BASTOS, 1996; BORGES, 2000; BARROS FILHO, 2007). Para além das suas competências ao nível do cruzamento de informação, é ainda de salientar a possibilidade de tratamento de dados a diferentes escalas, a possibilidade de executar análises espaciais e temporais essenciais (quer para a avaliação, como para a explicação de padrões e tendências de procura/utilização), a construção de cenários e a elaboração de modelos prospectivos de auxílio ao planejamento de ações (DOMINGUES, FRANÇOSO, 2008). Os SIG são, por isso, ferramentas fundamentais para a identificação e tratamento de indicadores de contexto (ROCHAet al., 2000; SANTANA, 2005).

Em termos de dados, o SIG contêm duas bases de dados intimamente integradas: espacial (locacional) e de atributos (estatística). A primeira contém informação, sob a forma de coordenadas digitais, usualmente proveniente de mapas ou de detecção remota (sob a forma de pontos, linhas ou polígonos). A base de dados dos atributos contém informação acerca das características ou qualidades da configuração espacial. Utilizando ambas as bases de dados, os SIG podem ser usados para investigar questões acerca da localização, das condições, das tendências ou dos percursos e padrões e, ainda, para fazer simulações (SANTANA, 2005).

A necessidade de gerir o espaço urbano de forma integrada e a preocupação com a qualidade de vida do organismo cidade têm levado alguns municípios urbanos a interessarem-se cada vez mais pelo uso do SIG (BORGES, 2000). No estudo realizado por BUDIC (1994), relativo à eficácia dos SIG no planejamentolocal ${ }^{4}$, avaliou-se os benefícios resultantes dos SIG em dois âmbitos: eficácia operacional e eficácia na tomada de decisão. A

${ }^{4}$ Através de um inquérito realizado em 125 municípios, em 4 Estados dos Estados Unidos da América. 
autora concluiu que em relação à eficácia operacional, a experiência de trabalho com os SIG aumenta a qualidade e a quantidade de dados disponíveis para o processo de planejamento(relacionado com melhorias na acessibilidade, na precisão e na disponibilidade dos dados). Por outro lado, a utilização dos SIG para análises de dados, incrementa a eficácia na tomada de decisão (associado a progressos na comunicação da informação e confiança nas análises realizadas com os SIG).

De facto, os SIG são ferramentas importantes na gestão pública, indispensáveis numa administração eficiente, auxiliando a tomada de decisão e o planejamento estruturado e saudável das áreas urbanas e do seu crescimento (ALMEIDA et al., 2007). O melhor conhecimento da realidade urbana permite levar a cabo intervenções com maior segurança, a monitorização dos impactos resultantes das políticas públicas, a disponibilização de informação a todos os sectores da administração local, propiciando, por um lado, uma maior eficiência na realização dos trabalhos, e, por outro lado, oferecendo à população uma prestação de serviços com qualidade, transparência e acessibilidade (DOMINGUES, FRANÇOSO, 2008).

Vários são os autores que têm discutido o papel destas plataformas como vantagem estratégica para os processos de gestão e planejamentourbano e para a própria actuação na Saúde Urbana (SANTANAet al., 2008). CRONER, BROOME (1996:1961) referemque "GIS will have a profound impact on public health strategies involving surveillance, risk assessment, analysis, and the control and prevention of human disease".

No entanto, o papel a desempenhar por estes sistemas deverá ir mais além, olhando a saúde não restritamente associada à doença. BARCELLOS, BASTOS (1996:392-393) mencionam o facto de os SIG possibilitarem a incorporação de uma gama de variáveis, como a extensão, localização, tempo e características socioeconómicas, aos estudos de saúde, uma vez que, as informações ambientais e de saúde geradas permitem a identificação de variáveis que revelem a estrutura social, econômica e ambiental, do lugar, onde os riscos para a saúde estão presentes, possibilitando planear medidas de intervenção. Assim, a utilização dos SIG no planejamento em saúde possui vantagens não só na detecção dos seus determinantes, mas também na análise e apresentação visual de clusters (BARCELLOS, BASTOS, 1996), podendo influenciar o modo como as decisões sobre o espaço são tomadas (DOMINGUES, FRANÇOSO, 2008). 
Sendo a saúde um tema estratégico no seio dos processos de desenvolvimento, a intervenção, quer na sociedade quer no espaço cidade, apenas pode ser sustentada e efetiva se resultar de informação fidedigna, fiável, adequada e disponibilizada em tempo útil, tendo para isso contribuído o uso dos SIG (SANTANA et al., 2008). A identificação, medição e avaliação dos fatores ambientais/de contexto proporcionada pelos SIG é fundamental para a atuação na saúde permitindo intervir na qualidade de vida e no bem-estar de cada indivíduo e da comunidade, fazendo a diferença no processo de planejamento do ambiente urbano (BUDIC, 2000; SANTANAet al., 2008).

É interessante referir que o primeiro SIG surgiu precisamente do cruzamento de dados de saúde e contexto: o mapa de John Snow (1854), em que este representou as mortes por cólera em cerca de seis quarteirões da cidade de Londres e identificou o poço como possível local de contágio e a água contaminada como vector de contágio (EYLER, 2001). De facto, os SIG suportam a inter-relacão entre meio ambiente (físico e social) e os aspectos geográficos da epidemiologia, tendo em vista a promoção e prevenção da saúde (SANTANA, 2005). Adicionalmente, o sistema de referência espacial e temporal permite adicionar novos fatores de explicação dos fenómenos em avaliação: ambientais, sociais, comportamentais, tendo em conta o espaço e o tempo. Por exemplo, a análise das características da distribuição das doenças, da localização dos recursos de saúde (oferta e acesso/utilização), da localização de potenciais condições de risco, por exemplo, e, ainda, as respectivas variações no tempo são possíveis através da aplicação de um SIG. Podem ainda ser estudadas as desigualdades, não só em termos de resultados em saúde como também em termos de acesso e prestação de serviços e relacioná-las com a distribuição das características socioeconómicas da população em risco.

O objetivo deste artigo é demonstrar a aplicabilidade dos SIG na análise da componente saúde no âmbito dos instrumentos de gestão territorial ou estudos de planejamento do território, nomeadamente através da construção de indicadores que contribuam para identificar e avaliar o impacto das características do contexto na saúde da população.

Foi selecionada Amadora, como área de estudo, por ser um município da área Metropolitana de Lisboa com o rápido crescimento populacional e habitacional, destacando-se áreas com padrões espaciais reveladores de clusters de alta privação sociomaterial (baixa escolaridade, emprego não qualificado e desemprego masculino)(SANTANA et al., 2008). 


\section{MATERIAL E MÉTODOS}

Como estudo de caso apresentar-se-á o Municipio de Amadora (Portugal) e, em específico, os indicadores de contexto que os SIG permitiram construír. Foram aplicadas várias metodologias de análise espacial, nomeadamente de proximidade e análise multicritério.

O indicador de proximidade foi construído com base em três metodologias distintas:buffer, área de serviço e equipamento mais próximo. A primeira consiste na construção de faixas de zonamento, ou seja, em torno de um tema é aplicadauma zona de uma determinada dimensão. Este método foi utilizado para construção das áreas de proteção das ribeiras. A segunda consiste na construção de zonas de serviço, com recurso à rede viária, e através de coroas de tempo. O terceiro método, tal como o segundo, tem por base a teoria de grafos, consistindo no cálculo do tempo de deslocação em rede viária a todos os equipamentos e identificação do mais próximo (COSTAet al., 2010).

Como FERREIRA et al.(2004) referem, a análise multicritério parte da premissa de que uma decisão corresponde a uma escolha entre várias alternativas, sejam elas relacionadas com ações, localizações, ou qualquer outra temática, tentando conjugar os critérios, de acordo com um objetivo específico e de forma a alcançar uma base composta de suporte à decisão. Existem vários métodos de análise multicritério. Neste artigo apresentam-se dois exemplos que recorrem ao Método Booleano Simples (o Índice de Vulnerabilidade Socioambiental e o Índice de Prevenção do Crime através do Desenho e Ambiente Urbano). Este método consiste em categorizar os critérios em 0 e 1, isto é, "não responde ao critério" e "responde ao critério", respetivamente.

Os indicadores construídos no projecto de investigação“Planeamento Urbano Saudável. Desenvolvimento e aplicação de um modelo ao caso da Amadora"estãoapresentados de acordo com as recomendações da Direcção Geral da Saúde de Portugal para a integração e apreciação da componente Saúde Humana nos Planos Municipais de Ordenamento do Território (Circular informativa no 36/DA de 9 de Outubro de 2009), isto é,agrupados em três grupos principais: 1. Ambiente Físico, 2. Estilos de Vida e 3. Condições de Vida. 


\section{RESULTADOS}

\section{Estudo de Caso: Amadora}

Entre2004 e 2008 um grupo de investigadoras da Universidade de Coimbra avaliou as interrelações entre os aspectos do ambiente físico e social (determinantes contextuais) e as características da população (composicionais), concluindo que os resultados em saúde na Amadora dependem de quem se é e do local onde se vive (SANTANAet al., 2008).Este projecto de investigação, subordinado ao tema "Planeamento Urbano Saudável. Desenvolvimento e Aplicação de um modelo ao caso da Amadora", obteve financiamento da Fundação para a Ciência e Tecnologia.

A seleção da Amadora como área de estudo ficou a dever-se à sua localização privilegiada, na primeira coroa de expansão da Área Metropolitana de Lisboa (AML) (figura 1), e ao rápido crescimento populacional e habitacional, consequênciada forte atração da população, com evidentes repercussões nos níveis de bem-estar e saúde de quem aí vive. No contexto da AML, é na Amadora que se observam as maiores disparidades, sendo possível identificar territórios de alta e baixa privação sociomaterial (analfabetismo, emprego não qualificado e desemprego masculino)(figura 2).

\section{Os SIG na avaliação do Ambiente Físico}

A caracterização do ambiente físico tem como objetivo a avaliação das condições do meio envolvente (natural e construído) a que a população está exposta e a identificação dos aspectos criticos para o conforto e saúde da população. Segundo a Circular informativa no 36/DA de 9 de Outubro de 2009, deve ser desenvolvido o perfil climático e condições de conforto higrotérmico, identificação dos riscos naturais, recursos naturais, avaliação da rede de saneamento e do espaço público. No caso de estudo da Amadora, os SIG permitiram avaliar, entre outros, a vulnerabilidade e constrangimentos do ambiente físico. 
Foram aplicadas metodologias de proximidade, consulta de informação e análise multicritério (SANTANA et al., 2007a). O indicador de vulnerabilidade foi contruído com base nas seguintes condições: 1 . área de protecção de ribeiras, 2 . proximidade a linhas de alta e muito alta tensão, 3. proximidade a linhas de comboio, 4. declive do terreno superior a 10 e 5. espaços de gestão pública (RAN e REN). As metodologias de proximidade foram aplicados para a contrução das condições 1 a 3; a extracção da informação às condições 4 e 5. A análise multicritério foi a metodologia aplicada para construção do índice de vulnerabilidade. A agregação destas condições permitiu identificar níveis de vulnerabilidade, classificados em 3 classes - baixo (área afectada por 1 ou 2 condicionantes), médio (área afectada por 3 condicionantes) e elevado (área afectada por 4 ou 5 condicionantes).

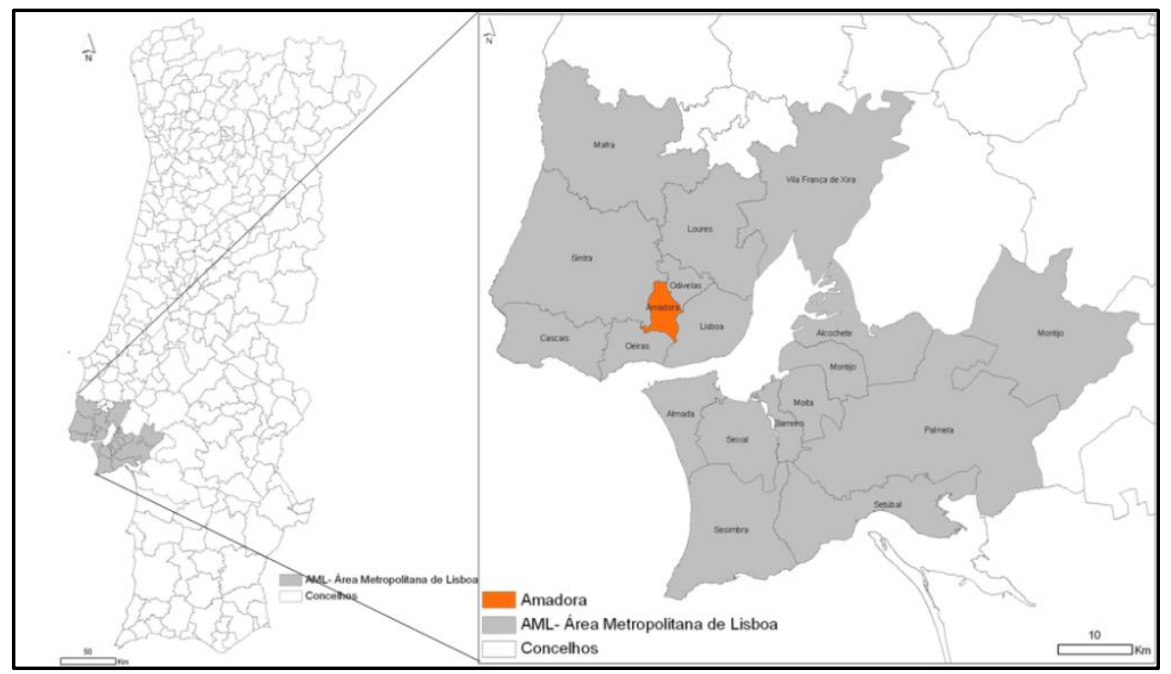

Figura 1. Localização do Município da Amadora na Área Metropolitana de Lisboa. 


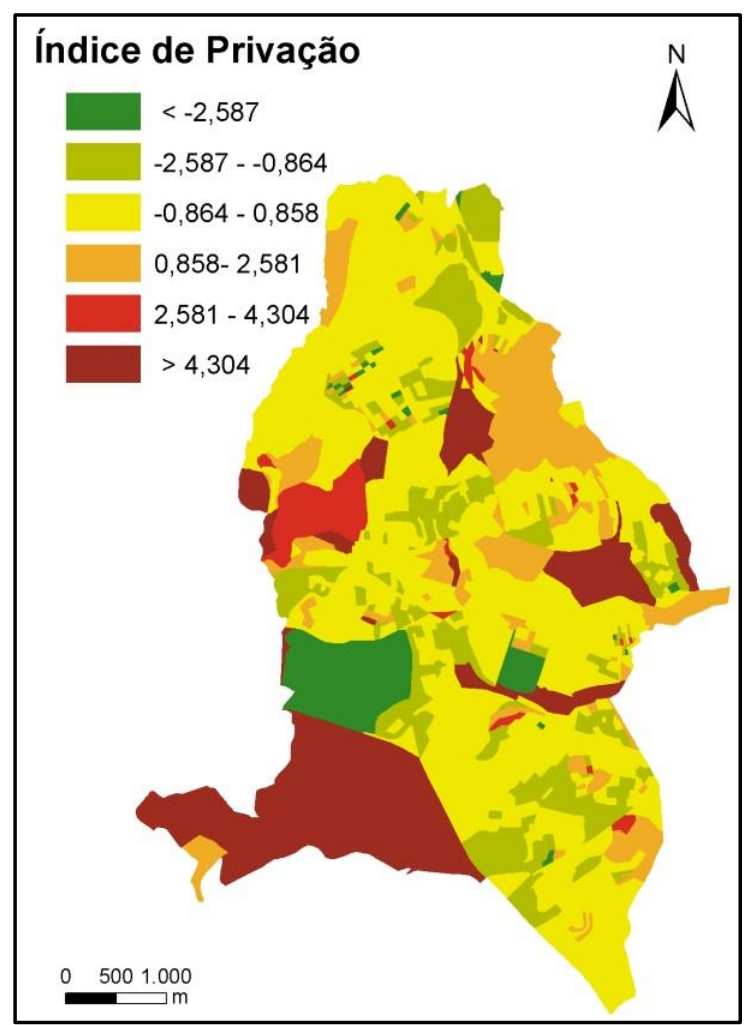

Figura 2. Índice de Privação Socio-material. Fonte: Santana et al, 2007a.

Da análise deste indicador verifica-se que mais de metade do território de Amadora encontra-se sob pelo menos uma condicionante física da vulnerabilidade e 4,5\% do território possui um risco médio ou elevado de vulnerabilidade (figura 3). Adicionalmente, verifica-se que $46 \%$ do edificado concelhio encontra-se em área vulnerável e as três classes de uso do solo relativas à construção e impermeabilização do solo - espaço urbano e equipamentos, comércio e indústria e vias de comunicação - ocupam quase $56 \%$ da área vulnerável. Estes valores são em parte explicados pelo processo de crescimento da cidade, determinado pela localização das estações e linha de caminho de ferro, o qual também explica a localização de áreas fortemente segregadas, social e espacialmente. De facto, 54,2\% dos núcleos degradados localizam-se na proximidade de linhas de alta tensão, 22,2\% em áreas de forte declive e $16 \%$ próximo de ribeiras. 


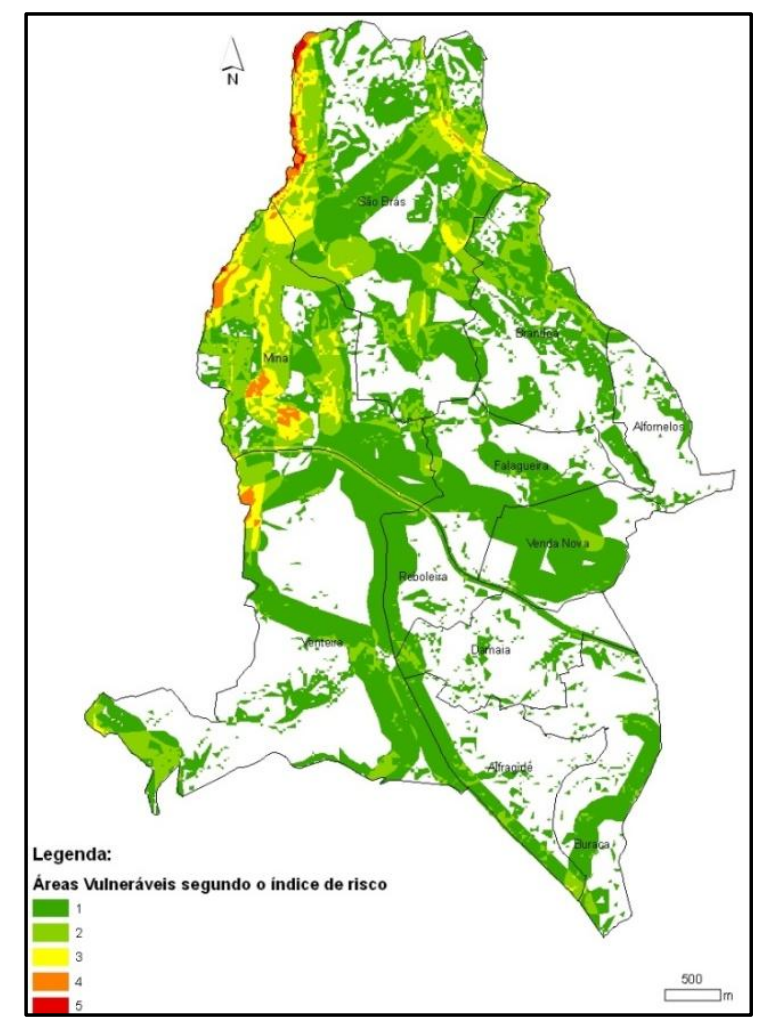

Figura 3. Áreas Vulneráveis segundo o Índice de Risco. Fonte: Santana et al, 2007a.

\section{Os SIG na avaliação das condições sociodemográficas e estilos de vida}

Avaliar quer a constituição sociodemográfica e a preponderância de cada grupo, quer as condições existentes para o desenvolvimento de estilos de vida saudáveis deve ser objetivo na avaliação do impacto dos instrumentos de gestão territorial na saúde da população, nomeadamente através da análise da estrutura sociodemográfica, hábitos e comportamentos ao nível de recreio e lazer e a coesão territorial do território.

O sentimento de insegurança foi um dos indicadores considerados de maior impacto na saúde da população do municipio da Amadora.

Para avaliação do sentimento de segurança/insegurança foram tomadas várias metodologias SIG, nomeadamente a geocodificaçãodos locais em que ocorreram crimes e a análise espacial na aplicação do índice de Prevenção do Crime através do Desenho e Ambiente Urbano - IPCDAU (SANTANA et al., 2007b). 
No município da Amadora observa-seuma incidência espacial da criminalidade de acordo com determinadas tipologias e identificam-se alguns hot-spots de criminalidade (figura 4). Estas áreas, genericamente, parecem sobrepor-se a áreas de níveis elevados de privação socioeconómica (figura 5). Quando analisada por tipologia de crime, verificou-se que onde existe maior privação existem menos crimes de furto em veículos e em residências e que os roubos por esticão são significativamente mais elevados nestas áreas ou na sua proximidade. Adicionalmente, a elevada prevalência de crimes que fragilizam mais as vítimas, porque as tornam mais inseguras (furto por esticão e roubo na via pública), é observada em áreas de níveis médios e altos de privação socioeconómica, enquanto os outros crimes, de natureza material mais relevante (furto de/em viaturas e furto em estabelecimentos), registam valores mais elevados em áreas de menor privação.

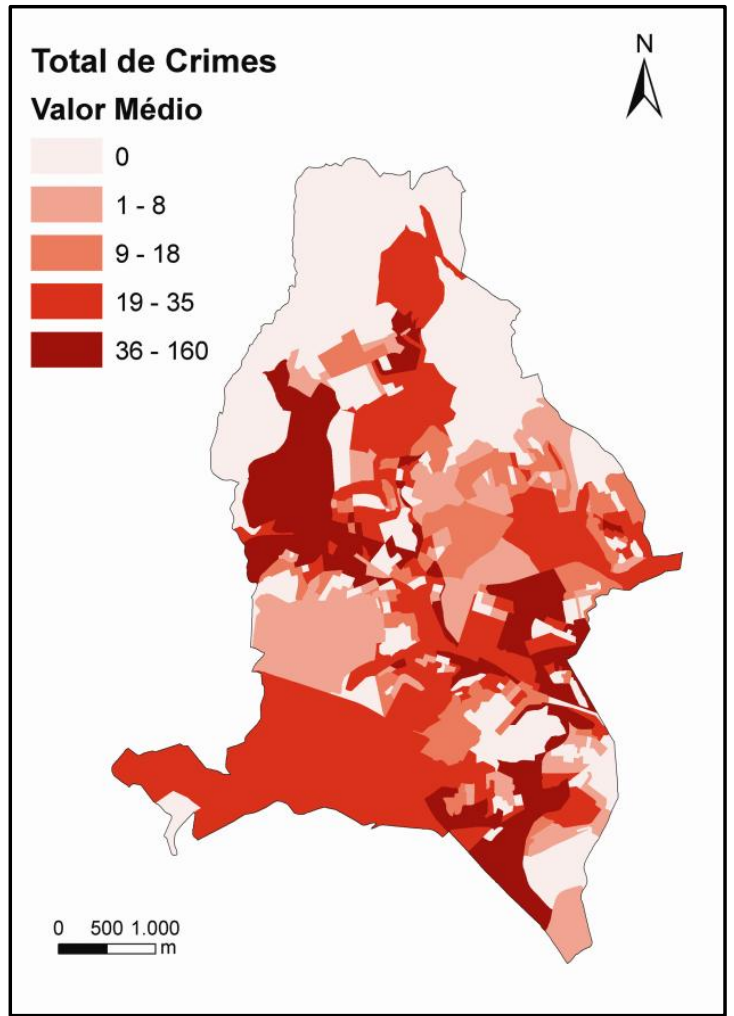

Figura 4. Crimes Participados (média 2004-06).

Fonte: PSP Amadora.

No entanto, aumentando a escala de análise verificamos que a criminalidade tem comportamentos diferenciados dentro dos clusters identificados anteriormente, sendo necessário procurar outras explicações para esta distribuição. De facto, quando analisamos a 
distribuição dos crimes por rua, verificamos que dentro de clusters de elevada criminalidade existem ruas em que a criminalidade é diminuta. Esta evidência conduziu a uma análise mais pormenorizada aplicando o índice de Prevenção do Crime através do Desenho e Ambiente Urbano - baseado no CPTED(Crime Pevention Through Environmental Design) a uma área da Amadora (figura 6). Esta permitiu concluir que os melhores resultados verificam-se a sul, mais próximo do caminho-de-ferro, correspondendo a uma área em que os campos visuais são mais abrangentes e com menos rotas, potenciais, de fuga. Os valores mais baixos de segurança nocturna foram verificados numa pequena área marcada por edifícios de materiais precários, má iluminação e com ambiente físico mal cuidado; oposto do que se verificou na maioria das ruas, onde, por exemplo, os níveis de iluminação se revelaram adequados. O problema identificado com maior abrangência territorial diz respeito à fraca ou inexistente presença de vigilantes naturais; não foi registado qualquer tipo de mobiliário urbano (bancos de repouso, esplanadas, quiosques, etc.) que permita aos utilizadores deste espaço permanecer nele por alguns momentos e poderem participar, embora que inconscientemente, no processo de vigilância natural. Por outro lado, e ainda no âmbito da falta de vigilância natural, foi verificado que há fraca relação entre o interior e o exterior dos edifícios: frequentemente as janelas do 1ำ andar estão "protegidas" por grades e os edifícios comerciais não têm montras com acesso visual directo para a rua, dificultando a relação entre o interior e o exterior.

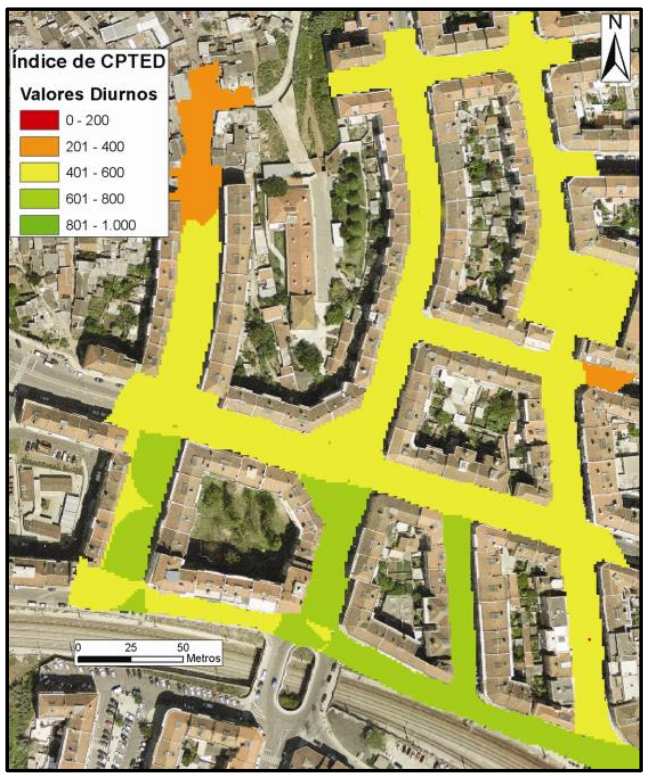

Figura 6. Valores diurnos de CPTED em ruas da

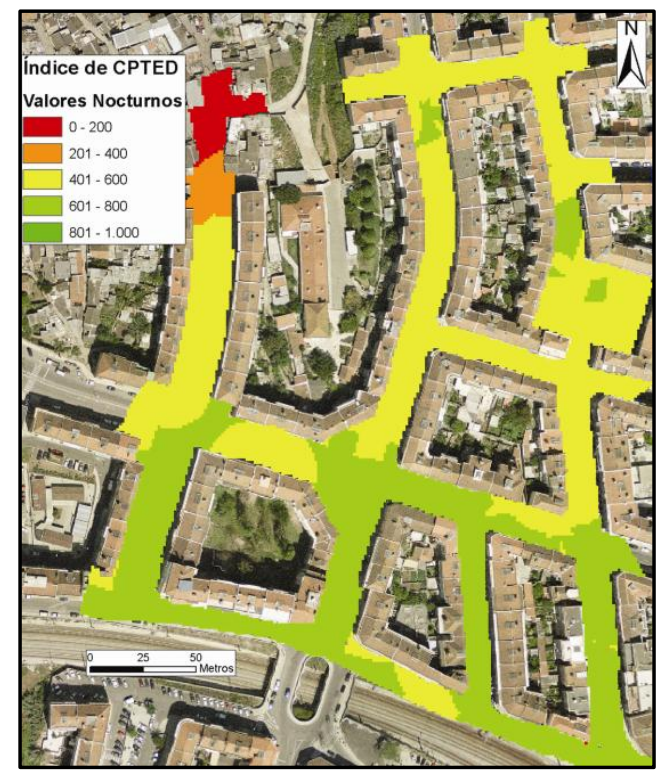

Figura 7. Valores nocturnos de CPTED em ruas 
Amadora.

Fonte: levantamento efectuado em 24 e 25 de Agosto de 2007. da Amadora.

Fonte: levantamento efectuado em 24 e 25 de Agosto de 2007.

\section{Os SIG na avaliação das condições de vida}

Quando o objetivo da análise passa pela avaliação das condições de base, relacionadas com oenquadramento da vida familiar e com a identificação de áreas e situações propiciadoras ao desenvolvimento de doenças e acidentes, é pertinente analisar a rede de cuidados de saúde, as características do parque habitacional, as condições sociais e os riscos em saúde pública.

Um exemplo de indicador que se pode construir é a acessibilidade geográfica aos equipamentos de saúde, a pé ou de transporte privado. Esta análise carece da construção de áreas de serviço às infraestruturas em questão (COSTA et al., 2010). No projecto da Amadora construíram-se áreas de serviço em redor dos centros e extensões de saúde, tendo em conta uma deslocação na rede viária a pé, e um tempo máximo de deslocação de 15 minutos.

Tendo em conta a população coberta por cuidados de saúde primários, segundo os níveis de acessibilidade, pode verificar-se que cerca de $29 \%$ percorre menos de 15 minutos, a pé, para aceder a um Centro de Saúde (figura 8 e 9). Ainda assim, verifica-se a existência de alguns bairros a norte que se localizam a mais de 30 minutos (velocidade de $3 \mathrm{~km} / \mathrm{h}$, percurso pedestre). No que diz respeito aos aspectos demográficos desta população, as crianças e jovens (grupo etário dos 0 aos 14 anos) estão afastados deste equipamento de saúde; apenas $23,8 \%$ está a menos de 15 minutos a pé. Em sentido oposto, os residentes com 65 e mais anos estão mais próximos dos Cuidados de Saúde Primários, quando comparados com a população total. 


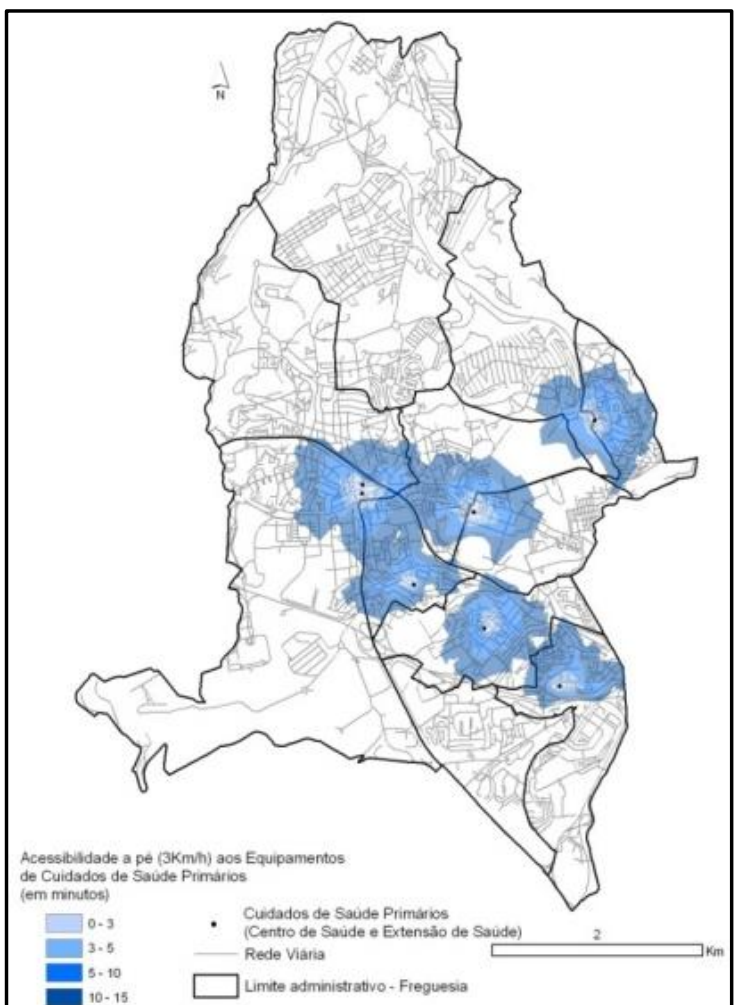

Figura 8. Acessibilidade geográfica (a pé) aos cuidados de saúde primários.

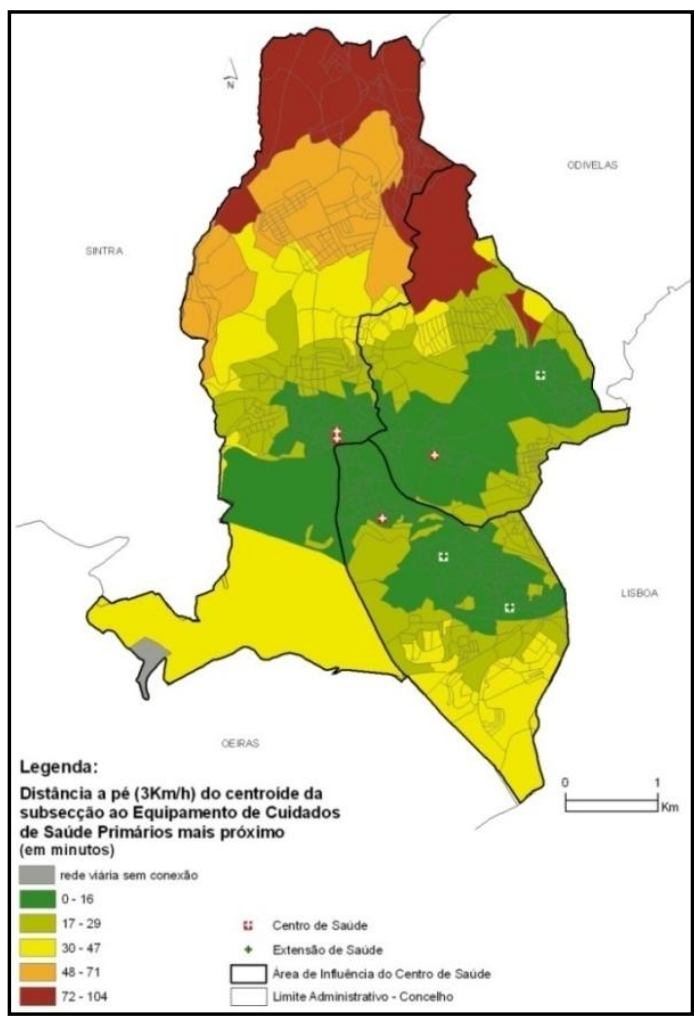

Figura 9. Distância a pé de cada bairro ao centro ou extensão de saúde mais próximo.

\section{DISCUSSÃO E CONCLUSÕES}

O projecto de investigação "Planeamento Urbano Saudável. Desenvolvimento e aplicação de um modelo ao caso da Amadora"apresentou, entre outros, como objetivos: 1 . contribuir para o avanço do conhecimento das questões relativas à saúde urbana; e 2. aplicar novas metodologias de análise e avaliação (nomeadamente de Sistemas de Informação Geográfica) do meio físico e social na saúde das populações e das comunidades (SANTANA et al., 2008). Deste projecto ressaltaram várias conclusões. A tarefa de identificar com precisão as características ambientais que podem influenciar a vida humana e a forma como podem ser medidas e monitorizadas, é um dos grandes desafios do planejamento urbano, dado que esse conhecimento é fundamental ao desenvolvimento de intervenções de planejamento 
saudável. Adicionalmente, os dados relativos às condições ambientais são de extrema utilidade na análise dos SIG na saúde, porque oferecem, fundamentalmente, a distribuição e possível(eis) correlação(ões) entre os fatores de risco e a saúde, numa distribuição espacial e temporal. Neste sentido, o uso de indicadores apropriados (materiais e imateriais), considerado um elemento central na operacionalização do conceito de cidade saudável, foi operacionalizado com recurso a metodologias SIG que permitiram identificar os fatores de contexto que têm maior impactono bem-estar e na saúde individual e comunitária, para além da sua medição, avaliação e monitorização.

$\mathrm{Na}$ literatura são vários os autores que reconhecem o papel do ambiente físico na determinação da qualidade de vida e da saúde,atribuindo ao lugar a capacidade de explicar os padrões de vida, saúde, doença e morte dos indivíduos (DIEZ-ROUXet al., 2001; CORBURN, 2004; SANTANA et al., 2009). De facto, o lugar faz já parte integrante da explicação dos resultados em saúde, através da verificação de associações entre áreas desfavorecidas e resultados em saúde (SANTANAet al., 2008).Os SIG aparecem como a ferramenta de conhecimento capaz de apoiar a explicaçãode como o lugar influencia a saúde individual e coletiva.

Contudo, os SIG não são uma panaceia e a sua utilização deve ser levada a cabo com cautela. Ou seja, por um lado os SIG têm capacidade para combinar dados de diferentes fontes e são particularmente indicados para a análise do impacto do meio ambiente na saúde humana e a detecção de problemas de ordem variada(ambientais, de oferta de recursos, etc.) associados directa ou indirectamente à saúde (doença) e, ainda,são essenciais enquanto suporte na decisão e planejamento de cuidados de saúde. Mas, por outro lado, para além das suas evidentes capacidades, os SIG têm também fraquezas e a possibilidade de utilizar dados relativos à saúde geo-referenciados não significa que os dados são aplicáveis ou utilizáveis sempre numa análise de SIG (SANTANA, 2005). Por exemplo, nos países desenvolvidos, onde os SIG são um importante instrumento em várias áreas científicas e também na da saúde, os erros e as omissões são o resultado de políticas públicas que respeitam a confidencialidade e privacidade da informação do serviço de assistência médica. Nos países em desenvolvimento, a informação é obsoleta ou incompleta, o que provoca erros e falsas conclusões.Adicionalmente, sem informação, principalmente informação geográfica, não é possível desenvolver um SIG e esta nem sempre está disponivel com a 
qualidade necessária (discrepância temporal, desiquilibrio entre o recolhido e o pretendido, mau preenchimento, falta de normalização, etc.). Esta questão condiciona as conclusões e, consequentemente, as decisões que se extraiam da análise. O mesmo ocorre com a escala de análise e metodologia SIG aplicada. Por exemplo, a proximidade pode ser avaliada através de buffers ou áreas de serviço e os resultados que advêm da sua aplicação são distintos. Ou seja, embora estes métodos se revistam de grandes potencialidades, o seu uso em saúde depende, de forma definitiva, da validade e da adequação dos dados. MARNOTO (1999) e SANTANA (2005) referiram, aliás, que dos componentes essenciais do SIG, a informação geográfica (pelo valor que tem, pelo tempo que demora a sua recolha e actualização e, ainda, pelo respectivo custo) e a inteligência humana (para gerir e controlar), são os que os que merecem maior reflexão na implementação de projectos SIG.

O SIG construído e utilizado sob uma concepção sistémica tende a tornar-se um laboratório do conhecimento sobre relações entre os elementos geográficos, constituindo-se como ponto de partida para novas aproximações teóricas (BUDIC, 2000). Daí a urgência de repensar este Sistema, tendo em conta a qualidade dos dados, a organização e estruturação da informação de caracterização geográfica, a caracterização das transformações, a actualização da informação e monitorização de espaços ou territórios e a avaliação e simulação dos efeitos de transformação de natureza espacial com vista ao fornecimento de elementos de decisão.

\section{REFERÊNCIAS BIBLIOGRÁFICAS}

ALCOFORADO, M. J.; ANDRADE, H.. Clima e Saúde na cidade. Implicações para o ordenamento. In: SANTANA, P. (coord.). A Cidade e a Saúde. Coimbra: Edições Almedina, 2007. p.99-118.

ALMEIDA, F.; COSTA, G.; NASCIMENTO, R.; LEITE, V.; LOBÃO, D.; GENESTRA, M.; OSÓRIO, T.; CARELLI, F.. Geoprocessamento no Trato de Controle de Características Urbanas.Cadernos UniFOA. mar. 2007. Disponível em: http://web.unifoa.edu.br/cadernos/edicao/03/21.pdf. Acesso em: 30 de maio. 2007. 
BARCELLOS, C.; BASTOS, F.. Geoprocessamento, ambiente e saúde uma união possível?. Cadernos de Saúde Pública, Rio de Janeiro v.12, n.3, p.389-397. 1996.

BARROS FILHO, M.. Geoprocessamento no Planejamento e na Gestão de Cidades". Annals of the I Congresso Nacional de Desenvolvimento Regional (CONDER), Caruaru. 2007.

BARTON, H.; TSOUROU, C..Healthy Urban Planning. A WHO guide to planning for people. WHO/ROE. Londres: Spon Press, 2000.

BORGES, K.. A Gestão Urbana e as Tecnologias de Informação e Comunicação. InformáticaPública. v.2, n.2, p.17-24. 2000.

BUDIC, Z..Geographic Information Science Implications for Urban and Regional Planning.Urisa Journal. v.12, n.2, p.81-93. 2000.

BUDIC, Z..Effectiveness of Geographic Information Systems in Local Planning.Journal of the American Planning Association.v.60, n.2, p.244-263. 1994.

CORBURN, J..Confronting the Challenges in Reconnecting Urban Planning and Public Health.American Journal of Public Health.v.94, n.4, p. 541-546. 2004.

COSTA, C.; SANTANA, P.; SANTOS, R.; LOUREIRO, A., 2010. Pre-School Facilities and catchment area profiling: a planning support method. Lecture Notes on Geoinformation and Cartography. v.0, p. 97-117. 2010.

CRONER, C.; BROOME, J.. Geographic Information Systems (GIS): New perspectives in Understanding Human Health and Environmental Relationships. Statistics in Medicine.v.5, p.1961-1977. 1996.

DIEZ-ROUX, A.; MERKIN, S.; ARNETT, D.; CHAMBLESS, L.; MASSING, M.; NIETO, J.; SORLIE, P.; SZKLO, M.; TYROLER, H.; WATSON, L.. Neighborhood of residence and Incidence of Coronary Disease.The New England Journal of Medicine, v.345, p.99-136. 2001. 
DOMINGUES, C.; FRANÇOSO, M.. SIG na Gestão Pública: Análises e Desafios de uma Implantação. Livro de Actas 5o Congresso Luso-Moçambicano de Engenharia/2을 Congresso de Engenharia de Moçambique, 2 a 4 de Setembro, p.1-9. 2008.

EYLER, J.M..The changing assessments of John Snow's and William Farr's cholera studies.Präventivmed, v.46, p.225-232. 2001.

FERREIRA, J.; ROCHA, J.; TENEDÓRIO, J.; SOUSA, P..Ensaio de Delimitação de Corredores Verdes na Área Metropolitana de Lisboa. 2004. Disponível em: http://www.academia.edu/3111159/Ensaio_de_Delimitacao_de_Corredores_Verdes_na_Ar ea_Metropolitana_de_Lisboa. Acesso em: 30 de abril. 2004.

GUIMARÃES, R.. Saúde Urbana: velho tema, novas questões. Terra Livre. São Paulo, n.17, p.155-170. 2001.

HOU, F.; MYLES, J..Neighbourhood inequality, neighbourhood affluence and population health.Social Science \& Medicine, v.60, p. 1557-1569. 2005.

KEMM, J..Health impact assessment and Health in all policies.In:STAHL, T.; WIMAR, M.; OLLILA, E.; LAHTINEN, E.; LEPPO, K. (Eds.). Health in All Policies: Prospects and potentials. Ministry of Social Affairs and Health. 2006.p.189-208.

LOCHNER, K., KAWACHI, I., BRENNAN, R., BUKA, S..Social Capital and Neighbourhood Mortality Rates in Chicago.Social Science \& Medicine. v.56, p.1797-1805. 2003.

MARNOTO, J.. Sistemas de Informação Geográfica. Expresso (Sistemas de Iformação Geográfica - Dossiers Promocionais), v.1387, p.18-19. 1999.

MEDRONHO, R.. Geoprocessamento e Saúde, uma Nova Abordagem do Espaço no Processo Saúde Doença. Rio de Janeiro: FIOCRUZ/CICT/NECT. 1995.

MUÑOZ, F..L'espaiurbà i la salut: una visió histórica". In:NOGUE, J. ; PUIGBERT, L. ; BRETCHA, G. (Eds.). Paisatge i salut. Barcelona: Observatori del Paisatge de Catalunya. 2008. p.79-115. 
PARTIDÁRIO, M.R.; JESUS, J.. Fundamentos da Avaliação do Impacte Ambiental. Lisboa: UniversidadeAberta, 2003.

PORTER, D..Health, Civilization and the State: A History of Public Health from Ancient to Modern Times. New York: Routledge. 1999.

RIBEIRO, M.. Sistema de Informação Geográfica como Sistema de Apoio à Decisão no Planeamento Urbano de Castelo Branco. Dissertação de Mestrado.Instituto Superior Técnico. 2001.

ROBERT, A.; REITHER, N. A multilevel analysis of race, community disadvantage, and body mass index amaong adults in the US. Social Science \& Medicine.v.59, p. 2421-2334. 2004.

ROCHA, A.; OKABE, I.; MARTINS, M.; MACHADO, P.; MELLO, T.. Qualidade de vida, ponto de partida ou resultado final?. Ciênc. Saúde Coletiva. v.5, n.1, Rio de Janeiro, p. 63-81. 2000.

SALVEMINI, M.. Urban planning and GIS: an opposed marriage. Livro de Actas Second European GIS Education Seminar, Budapest, Hungary. 2000.

SANTANA, P.. Geografias da Saúde e do Desenvolvimento. Evolução e Tendências em Portugal. Coimbra: Edições Almedina, 2005.

SANTANA, P.; NOGUEIRA, H.; COSTA, C.; SANTOS, R.. Identificação das vulnerabilidades do ambiente físico e social na construção da Cidade Saudável. In: SANTANA, P. (coord.). A Cidade e a Saúde. Coimbra: Edições Almedina, 2007a. p.165-180.

SANTANA, P.; ROQUE, N.. A (In)Segurança na Amadora. In: SANTANA, P. (coord.). A Cidade e a Saúde. Coimbra: Edições Almedina, 2007C. p.181-196.

SANTANA, P.; SANTOS, R.;NOGUEIRA, H..The link between local environment and obesity: a multilevel analysis in the Lisbon Metropolitan Area, Portugal. Social Science\& Medicine,68(4) 2009, p. 601-609.

SANTANA, P.. Por uma Cidade Saudável. In: JANUS - Publicação conjunta do Jornal Público e Universidade Autónoma de Lisboa. 2009. 
SANTANA, P., SANTOS, R., COSTA, C. E LOUREIRO, A.. Pensar Amadora Cidade Saudável e

Activa. 3o Prémio de Reconhecimento da Rede Portuguesa de Cidades Saudáveis (policopiado). 2008.

VAN LETHE, F.; BRUG, J.; MACKENBACH, J.. Neighbourhood Inequalities in Physical Inactivity: The role of Neighbourhood Atractiveness, Proximity to Local Facilities and Safety in the Netherlands. Social Science \& Medicine. v.60, p. 763-775. 2005.

VASCONCELOS, J.; VIEIRA, R.. Conforto Bioclimático da Amadora.Contributo para o Planeamento Saudável. In: SANTANA, P. (coord.). A Cidade e a Saúde. Coimbra: Edições Almedina, 2007. p.197-218.

VEENSTRA, G.; LUGINAAH, I.; WAKEFIELD, S.; BIRCH, S.; EYLES, J.; ELLIOT, S.. Who you know, where you live: social capital, neighbourhood and health. Social Science \& Medicine.v.60, 2005.p. 2799-2818.

WEINSTEIN, M...Health in the city. New York: Pergamon Press Inc. 1980.

WHITFORD, V.; ENNOS, A. R.; HANDLEY, J. F.. City form and natural process - indicators for the ecological performance of urban areas and their application to Merseyside, UK.Landscape and Urban Planning.v.57, n.2, p.91-103. 2001.

WILSON, K.; EYLES, J.; ELLAWAY, A.; MACINTYRE, S.; MACDONALD, L.. Health status and health behaviours in neighbourhoods:a comparasion of Glasgow, Scotland and Hamilton, Canada. Health \& Place.v.16, p. 331-338. 2010. 